\title{
ORIGINAL RESEARCH \\ Percutaneous Embolization of Carotid Paragangliomas Using Solely Onyx
}

I. Wanke

M.C. Jäckel

S. Goericke

V. Panagiotopoulos

U. Dietrich

M. Forsting
BACKGROUND AND PURPOSE: Paragangliomas are highly vascularized usually benign neoplasms arising from nerve tissue. Endovascular preoperative embolization is used to facilitate surgery but is often not complete, due to tiny feeding arteries not feasible for selective catheterization. Our purpose was to evaluate angiographic and clinical outcome using Onyx for percutaneous glomus tumor embolization.

MATERIALS AND METHODS: A consecutive series of 4 patients with 6 paragangliomas located at the bifurcation of the common carotid artery were treated with percutaneous embolization using Onyx as the sole embolic material.

RESULTS: Complete devascularization of the 6 paragangliomas was achieved using a percutaneous embolization technique with Onyx as a sole agent, combined with an endovascular microballoon that offered protection of the internal carotid artery.

CONCLUSIONS: Further documentation is necessary to prove the higher grade of devascularization of paragangliomas with Onyx compared with other embolic material and the associated potential to facilitate surgery. Nevertheless, Onyx seems to be safe when used percutaneously due to its lavalike pattern flow and its controllable properties, allowing slow tumor bed penetration.
$\mathbf{P}$ aragangliomas are rare but most often benign neoplasms of the head and neck region arising from sympathetic or parasympathetic nerve tissue. These autonomous gangliomas are highly vascularized and characteristically have blood vessel and nerve involvement. Occasionally malignant degeneration has been reported. Traditionally, surgical resection of the tumors is performed, but substantial bleeding during surgery may occur. Endovascular preoperative embolization has been proved to facilitate surgery due to elimination of hypervascularity and tumor pulsation. Endovascular embolization by using polyvinyl alcohol particles and polymerized glue ( $n$-butyl cyanoacrylate $[n$-BCA $]$ ) via the arterial route is often not complete because tiny feeding arteries are not feasible for selective catheterization. A percutaneous approach to the lesion by using ethylene-vinyl alcohol copolymer (Onyx; ev3, Irvine, Calif), a relatively new embolic agent, might be a better solution for obliterating the entire tumor bed and, therefore, optimize surgery.

We present our results of complete devascularization of 6 paragangliomas located at the bifurcation of the common carotid artery by using a percutaneous embolization technique with Onyx as a sole agent, combined with endovascular microballoon protection of the internal carotid artery (ICA).

Received December 16, 2008; accepted after revision February 25, 2009.

From the Institute of Diagnostic and Interventional Radiology and Neuroradiology (I.W. S.G., V.P., M.F.), University of Essen, Essen, Germany; Department of Neuroradiology (I.W.), Klinik Hirslanden, Zürich, Switzerland; Department of Oto-Rhino-Laryngology (M.C.J.), University Hospital of Essen, Essen, Germany; Departments of Neurosurgery and Interventional Neuroradiology (V.P.), University of Patras, Patras, Greece; and Department of Neuroradiology (U.D.), Krankenanstalten Gilead, Bielefeld, Germany.

Please address correspondence to Isabel Wanke, MD, PhD, Department of Diagnostic and Interventional Radiology and Neuroradiology, University Hospital of Essen, Hufelandstr 55, 45147 Essen, Germany; e-mail: isabel.wanke@uni-due.de

DOl 10.3174/ajnr.A1601

\section{Materials and Methods}

\section{Patients}

A consecutive series of 4 patients with 6 carotid body paragangliomas was treated with percutaneous embolization by using Onyx as the sole embolic material. The age of the patients ranged between 32 and 67 years with a mean of 44.3 years. The largest tumor was $5.0 \mathrm{~cm}$, with mean diameter of $3.5 \mathrm{~cm}$. None of the patients had neurologic symptoms; specifically none of them demonstrated cranial nerve palsies. All patients noted palpable masses at the neck. In 2 patients, a familial history of glomus tumors was known.

\section{Illustrative Case}

A 63-year-old male patient presented with 2 carotid body paragangliomas, each located typically at the extracranial bifurcation on both sides. The patient was asymptomatic, without any evidence of cranial nerve dysfunction, but he could feel and palpate the masses in his neck bilaterally. Diagnostic work-up included contrast-enhanced MR imaging and diagnostic digital subtraction angiography (DSA), with injection of both common carotid arteries (Fig $1 A,-B$ ). The tumors measured $5 \times 4.5 \mathrm{~mm}$ on the right side and $3.9 \times 2.2 \mathrm{~mm}$ on the left side, according to MR imaging. DSA showed high vascularity of both tumors with arterial supply from feeders originating from the external and internal carotid arteries (ECA and ICAs). Before surgical extirpation, both tumors were percutaneously embolized by using Onyx, a liquid dissolved in dimethyl-sulfoxide (DMSO) (Fig $1 C,-D)$. In this patient, both tumors were treated at 2 different time points with an interval of 4 months.

\section{Technique}

The procedures were performed with the patient under general anesthesia to avoid movement and pain due to Onyx injection. A transfemoral approach was used to insert a $6 \mathrm{~F}$ guiding catheter into the common carotid artery to allow positioning of a protective microballoon into the proximal ICA and for intraprocedural angiographic control of Onyx distribution into the tumor bed ("casting"). After choosing the best working projection by using a biplane angiography unit (Infinix NB; Toshiba Medical Systems, Tokyo, Japan), a road- 

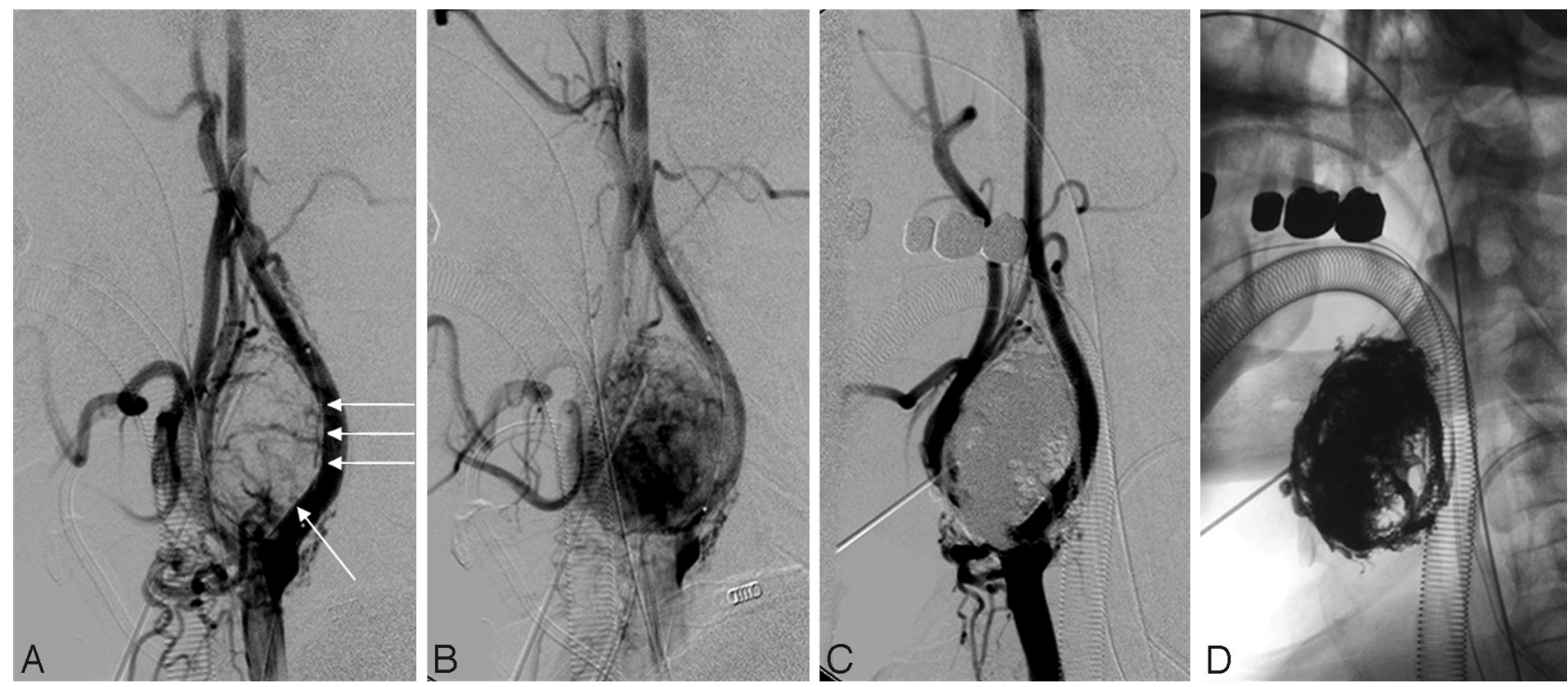

Fig 1. Lateral common carotid artery injection. $A$ and $B$, Early $(A)$ and late phase $(B)$ images show a carotid paraganglioma in loco typico, with arterial supply from the ascending pharyngeal artery, proximal tiny vessels from the ECA, and the proximal ICA (arrows). C, Immediately after embolization, the tumor was devascularized. D, The unsubtracted image shows the Onyx cast in the tumor bed.
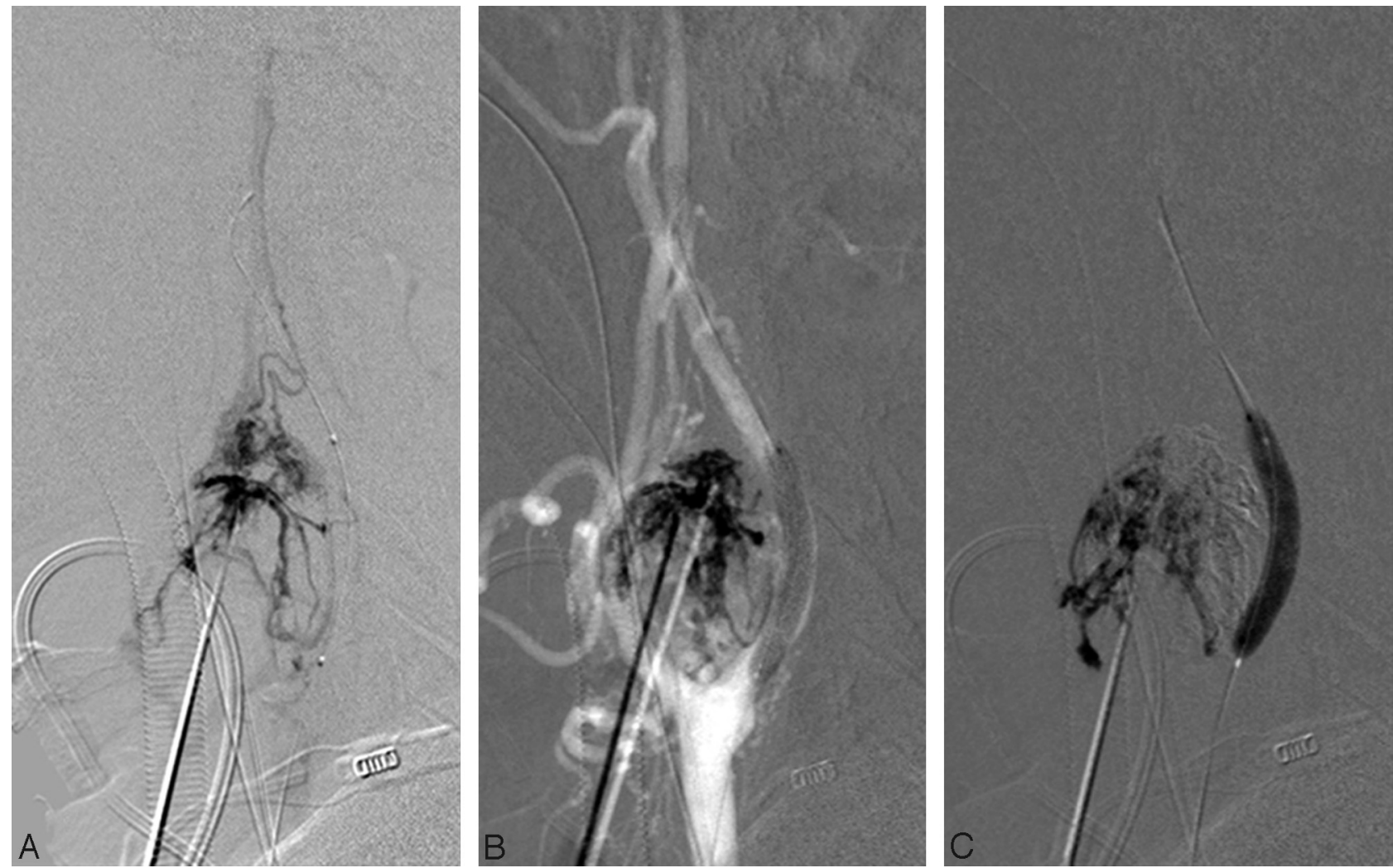

Fig 2. Embolization technique after percutaneous tumor puncture. A parenchymogram was obtained $(A)$, and injection of Onyx was started by using a roadmap technique ( $B$ ) with inflation of the microballoon $(C)$ to protect the lumen of the ICA.

mapping technique with 2 projections through the common carotid artery was achieved to facilitate tumor puncture percutaneously. A microballoon (HyperGlide 4/30 mm; ev3) was then inserted through the guiding catheter and placed at the proximal ICA to protect the vessel lumen temporarily during injection of Onyx. Percutaneous tumor puncture was performed with a 21-gauge spinal needle (Terumo, Tokyo, Japan). After puncture, a parenchymogram (Fig 2A) was ob- tained to ascertain correct intratumoral needle position and exclude extravasation of contrast material to normal parenchyma.

After flushing the delivery system (a short tubing with a diameter of $1.2 \mathrm{~mm}$ from Braun, Melsungen, Germany; and the Terumo spinal needle) with DMSO to avoid precipitation, slow injection of Onyx 18 was started under continuous fluoroscopy (Fig 2B). No premature precipitation in the delivery system was noted. A blank roadmap (Fig 


\begin{tabular}{|c|c|c|c|c|c|c|c|c|c|}
\hline \multicolumn{10}{|c|}{ Patient demographics and carotid body tumor characteristics } \\
\hline $\begin{array}{l}\text { Patient } \\
\text { No. }\end{array}$ & Sex & $\begin{array}{c}\text { Tumor } \\
\text { No. }\end{array}$ & $\begin{array}{c}\text { Tumor } \\
\text { Location }\end{array}$ & $\begin{array}{l}\text { Tumor } \\
\text { Size } \\
(\mathrm{cm})^{*}\end{array}$ & $\begin{array}{l}\text { Origin of } \\
\text { Feeding } \\
\text { Pedicles }\end{array}$ & $\begin{array}{c}\text { No. } \\
\text { Punctures }\end{array}$ & $\begin{array}{c}\text { Onyx } \\
\text { Volume } \\
\text { (mL) }\end{array}$ & $\begin{array}{l}\text { Duration of } \\
\text { Injection } \\
\text { (min) }\end{array}$ & Devascularization \\
\hline \multirow[t]{2}{*}{1} & $\mathrm{~F}$ & 1 & Left & 2 & ICA/ECA & 1 & 3.0 & 15.0 & Total \\
\hline & & 2 & Right & 3.6 & ICA/ECA & 2 & 3.1 & 19.0 & Total \\
\hline \multirow[t]{2}{*}{2} & M & 3 & Left & 5 & ICA/ECA & 2 & 9.1 & 26.4 & Total \\
\hline & & 4 & Right & 3.0 & ICA/ECA & 1 & 3.9 & 16.8 & Total \\
\hline 3 & $\mathrm{~F}$ & 5 & Left & 3.9 & ICA/ECA & 1 & 5.2 & 21.6 & Total \\
\hline 4 & $\mathrm{~F}$ & 6 & Left & 3.5 & ICA/ECA & 1 & 4.2 & 17.9 & Total \\
\hline
\end{tabular}

Note:-ICA indicates internal carotid artery; ECA, external carotid artery

* Longest diameter.

2C) was helpful to illustrate potential retrograde injection of Onyx into the ICA through small feeders arising from the ICA. The microballoon was temporarily inflated only if Onyx came close to the vessel wall of the ICA, to avoid retrograde injection of the embolic agent into the lumen or if the Onyx cast overlay the ICA and hindered visibility of the vessel lumen. The maximal inflation time of the microballoon was 3 minutes (collaterals were checked beforehand). Embolization was stopped when the control angiogram through the common carotid artery showed complete tumor obliteration (Fig $1 C,-D$ ). The duration of the embolization procedures lasted for a mean of 56.6 minutes (range, 45-70 minutes). Arterial angiography was analyzed to determine the degree of devascularization. Detailed neurologic assessment was performed after embolization and after surgery. Surgery was performed within 5 days after each percutaneous embolization. Duration of surgery was a mean of 151 minutes (range, 80-280 minutes). The amount of blood loss and the ease of extirpation were determined intraoperatively. The specimen was sent to pathology.

In the illustrative case, 1 needle position was sufficient for the first paraganglioma; in the second tumor, 2 needle positions were necessary for embolization. As a result, complete tumor embolization could be achieved on both sides by using Onyx as a sole embolic material. The main feeding arteries (ICA, ECA, and ascending pharyngeal artery) remained open, whereas the tiny feeding arteries arising from those vessels could be occluded.

Surgery was performed by using magnifying loops. Both tumors were removed by bipolar and sharp dissection in a craniocaudal fashion. The dissection was done in the subadventitial plane. Intraoperative blood loss was minimal and amounted to approximately $250 \mathrm{~mL}$ (right) and $150 \mathrm{~mL}$ (left). The operating times were 3 hours $50 \mathrm{~min}$ utes and 4 hours 40 minutes, respectively. Complete tumor removal was achieved as en bloc removal and was easily performed; no blood transfusions were necessary.

\section{Results}

All tumors could be completely embolized by using 1 singleneedle position in 4 tumors and 2 needle positions in 2 tumors (Table). Extirpation en bloc without significant loss of blood was performed within 5 days after embolization in all patients. Diagnosis of paragangliomas was confirmed histologically in all patients. No adverse events occurred during the interventional procedures; no clinical complications were noted. Only 1 patient noted some pain while pressing her finger on the tumor after embolization. The patients did not show any neurologic symptoms after either the percutaneous embolization or surgical tumor removal.

\section{Discussion}

Paragangliomas are highly vascularized tumors, which might lead to a considerable blood loss during surgical extirpation. The potential invasiveness of an embolization must be justified by providing a clear advantage during surgery. Tumor devascularization reduces intraoperative blood loss, thus resulting in better defining the planes of dissection and simplifying total tumor resection.

Several techniques concerning glomus tumor embolization via the endovascular route (arterial, venous, or combined) or through direct percutaneous puncture have been previously described in the literature. Endovascular embolization was shown to facilitate surgical tumor removal and diminish the need for red blood cell transfusions. ${ }^{1-3}$ Injection of Onyx through a combined arterial and venous route has also been reported to offer complete embolization of a paraganglioma. ${ }^{4}$

A percutaneous approach by using liquid embolic material such as cyanoacrylate glue, which has been previously described, was shown to be safe and effective and might have the advantage of obliterating the tumor bed in contrast to the endovascular tumor approach. ${ }^{5-7}$ Nevertheless, there are potential risks of acute displacement of glue into brain-supplying arteries during injection, ${ }^{8}$ and even delayed migration has been described. ${ }^{9}$ Extreme caution must be used due to "dangerous" collaterals and retrograde filling of small vessels arising from the ICA and vertebral artery.

We report 4 patients treated for 6 carotid paragangliomas supplied by tumor vessels arising from the ECA and ICA. The tumors were embolized by direct puncture of the tumor, and complete obliteration could be achieved by using Onyx as the sole embolic agent. Although percutaneous tumor embolization with Onyx has already been described in the literature, ${ }^{10}$ it was used in combination with other embolic material such as particles administered by the endovascular route. In this case, tumor obliteration was not complete. Recently Elhammady et $\mathrm{al}^{11}$ reported a single case of direct percutaneous embolization of a carotid body tumor with Onyx and confirmed the ease of access to the tumor vascularity by direct percutaneous tumor puncture, resulting in complete obliteration. A typical finding in carotid paragangliomas is the supply from extremely small vessels originating from the proximal part of the ICA and ECA, as in the case illustrated above (Fig 1A).

These tiny vessels cannot be selectively catheterized by endovascular techniques. With a percutaneous approach, complete obliteration even of those small vessels can be achieved. 
However, because direct intratumoral injection of liquid polymerizing agents may result in retrograde filling of those vascular channels, it bears the risk of migration of embolic material into intracranial arteries. Therefore, precautions to avoid such circumstances should be undertaken, even using a controllable slow-migrating embolic agent like Onyx. In our case, a nondetachable microballoon was inserted and temporarily inflated in the proximal ICA at the origin of the tiny tumor branches. The use of Onyx as a liquid embolic agent proved to be safe because the injection rate is very slow; because Onyx behaves like lava flow, the velocity of Onyx spread is much more controllable than that using conventional acrylates, especially if arteriovenous shunt surgery is present in the tumor.

Onyx is known and widely accepted for embolization of intracranial arteriovenous malformations. The flow characteristics of Onyx in contrast to conventional liquid embolic materials like $n$-BCA are optimal for tumor embolization by direct puncture, demonstrated in our series of 6 paragangliomas. Determining angioarchitecture by analyzing all feeding vessels plays a minor role when using Onyx. This embolic agent has the advantage of distributing slowly in an ante- and retrograde way to the entire tumor bed, even if it is supplied by different arteries. The fact that in most of our patients 1 needle position was sufficient underlines this fact. Regarding the degree of devascularization, direct puncture of the tumor with percutaneous embolization is considered superior to the endovascular approach. Tumor removal was considered easy due to devascularization and the elastic consistency of Onyx.

Further documentation is necessary to prove the higher grade of devascularization of paragangliomas with Onyx com- pared with conventional glue or other embolic material and the associated potential to facilitate surgery. Nevertheless, Onyx seems to be safe when used percutaneously due to its lavalike pattern flow and its controllable properties, allowing slow tumor-bed penetration. Onyx might be also an optimal embolic liquid to preoperatively treat paragangliomas percutaneously at more challenging locations at the skull base.

\section{References}

1. Valavanis A. Preoperative embolisation of the head and neck: indications, patient selection, goals, and precautions. AJNR Am J Neuroradiol 1986; 7:943-52

2. Murphy TP, Brackmann DE. Effects of preoperative embolization on glomus jugulare tumors. Laryngoscope 1989;99:1244-47

3. Persky MS, Setton A, Niimi Y, et al. Combined endovascular and surgical treatment of head and neck paragangliomas: a team approach. Head Neck 2002;24:423-31

4. Rimbot A, Mounayer C, Loureiro C, et al. Preoperative mixed embolization of a paraganglioma using Onyx. J Neuroradiol 2007;34:334-39

5. Casasco A, Herbreteau D, Houdart E, et al. Devascularization of craniofacial tumors by percutaneous tumor puncture. AJNR Am J Neuroradiol 1994; 15:1233-39

6. George B, Casasco A, Deffrennes D, et al. Intratumoral embolization of intracranial and extracranial tumors: technical note. Neurosurgery 1994;35:771-73, discussion 773-74

7. Abud DG, Mounayer C, Benndorf G, et al. Intratumoral injection of cyanoacrylate glue in head and neck paragangliomas. AJNR Am J Neuroradiol 2004;25:1457-62

8. Casasco A, Houdart E, Biondi A, et al. Major complications of percutaneous embolization of skull-base tumors. AJNR Am J Neuroradiol 1999;20:179-81

9. Krishnamoorthy T, Gupta AK, Rajan JE, et al. Stroke from delayed embolization of polymerized glue following percutaneous direct injection of a carotid body tumor. Korean J Radiol 2007;8:249-53

10. Quadros RS, Gallas S, Delcourt C, et al. Preoperative embolization of a cervicodorsal paraganglioma by direct percutaneous injection of Onyx and endovascular delivery of particles. AJNR Am J Neuroradiol 2006;27:1907-09

11. Elhammady MS, Farhat H, Ziayee H, et al. Direct percutaneous embolization of a carotid body tumor with Onyx. J Neurosurg 2009;110:124-27 